\title{
Carbon Nanotubes Enhanced Cellulose Nanocrystals Films with Tailorable Electrical Conductivity
}

Qingkai Meng and Ica Manas-Zloczower*

Department of Macromolecular Science and Engineering, Case Western Reserve University 2100 Adelbert Road, Cleveland, Ohio 44106, USA

Abstract: Cellulose nanocrystals (CNCs) and commercial multi-walled carbon nanotubes (CNTs) are used to prepare CNT enhanced CNC films at various CNT weight fractions via an aqueous suspension vacuum filtration method with the purpose of studying potential synergistic effects between the CNTs and the CNCs in hybrid nanoparticle networks. The addition of CNTs even at very low concentrations allows the use of vacuum filtration for CNC films. The hybrid films show superior tensile and promising electrical properties by comparison with the $\mathrm{CNC}$ film. CNT enhanced $\mathrm{CNC}$ films prepared with micro-size CNT aggregates in the absence of surfactant exhibit improved tensile properties by comparison with the $\mathrm{CNC}$ films, suggesting that the CNCs could help disperse and stabilize the CNTs in aqueous suspensions. A maximum in tensile properties is obtained at a CNT concentration of around $10 \mathrm{wt}$ \% with 6.26GPa modulus and 179.7MPa strength. Moreover the hybrid films are more ductile than the CNC film, resulting in improved tensile toughness. The surface electrical resistivity for the hybrid films can be tuned within a range from $10^{2}$ to $10^{11} \Omega /$ sq by manipulating the CNT weight fraction as well as the environmental humidity.

Keywords: cellulose nanocrystals, A. carbon nanotubes, A. hybrid composites, B. mechanical properties, B. electrical properties

* Corresponding author

E-mail address: ixm@case.edu (Ica Manas-Zloczower) 


\section{Introduction}

Carbon nanotubes (CNTs) have attracted extreme academic interests due to their unique carbon nanostructures and exceptional intrinsic properties [1, 2]. A Young's modulus of $1.28 \pm 0.59 \mathrm{TPa}$ was reported for multi-walled carbon nanotubes (MWCNTs) comparable to the in-plane modulus of 1.06TPa for graphite, the largest modulus of any known bulk material[3]. Such superlative mechanical properties are extremely attractive for CNT applications in high strength materials. In addition, CNTs also possess desirable electrical and thermal properties suitable for advanced functional material applications such as conductive composite materials and electrochemical devices [4-7]. Recently, another type of nanoparticles - cellulose nanocrystals (CNCs) have attracted lots of attention due to their intrinsic properties, the abundance and renewable resource for the raw materials, the biodegradability and the potential as next generation reinforcing nanofillers. Since CNCs are typically prepared by removing most of amorphous components (lignin and semicellulose) from cellulosic materials and leaving only the cellulose crystallites, the mechanical properties of $\mathrm{CNCs}$ are close to those of cellulose crystallites. The calculated Young's modulus along the chain axis is 167.5GPa for cellulose crystalline form I (native cellulose) and 162.1GPa for form II (regenerated cellulose) [8]. The experimentally measured elastic moduli for cellulose crystallites and cellulose nanocrystals cover a wide range from 50 to $220 \mathrm{GPa}$ with variation from cellulose source, preparation and measuring methods [9-13]. In addition to the attractive mechanical properties, another appeal of CNCs is their surface functionality which could be manipulated by different preparation/surface modification methods, for example, sulfate groups from sulfuric acid hydrolysis [14], carboxylate groups from TEMPO-mediated oxidation [15], acetate ester groups from acid-catalyzed 
esterification $[16,17]$ and preserved original hydroxyl groups from mechanical treatment [18]. Decorated with ionic charges on the surface, CNCs prepared via sulfuric acid hydrolysis and TEMPO-mediated oxidation can be easily dispersed in water due to electrostatic repulsion. This enables preparation of CNC films from $\mathrm{CNC}$ aqueous suspensions by solvent casting or membrane filtration.

Transparent cellulose nanofiber films with high gas barrier properties were prepared by filtrating TEMPO-oxidized cellulose nanofiber suspensions in water [19]. Ultrastrength cellulose nanopapers were manufactured from cellulose nanofibrils via membrane filtration method [20]. The properties of $\mathrm{CNC}$ films could be modified by incorporating other nanoparticles, for example, cellulose nanofiber/clay nanopapers extend the property range of cellulose nanopapers with improved fire retardant and gas barrier properties [21]. CNT enhanced CNC films are worthwhile for investigation because of expected properties from both nanoparticles and potential broad applications in the electronic field, such as electromagnetic interference shielding and electronic circuits [22].

To the best of our knowledge, few reports on CNT enhanced CNC films have been published so far. Salajkova et al. prepared nanopapers by a water based processing route from MWCNTs purified with nitric acid and hydrochloric acid and nanofibrillated cellulose (NFC) derived from a commercial softwood pulp by TEMPO-mediated oxidation [23]. A surfactant - nonylphenol POE-10 phosphate ester was required to stabilize purified MWCNTs in the MWCNT/NFC aqueous suspensions since the MWCNTs settled down without the surfactant. It was pointed out based on SEM and AFM images that the number of contacts between MWCNTs increases with increasing MWCNT content and at 9.1 wt.\% the MWCNTS form a continuous network; however, 
at the maximum MWCNT content used (16.7 wt.\%), agglomerates of MWCNTs were observed. Based on the mechanical properties results these authors concluded that MWCNTs could not provide positive contribution to the nanopapers because of the weak or no interaction between MWCNTs and NFC as well as the increased porosity with increasing CNT content. The electrical conductivity improved over more than 4 decades between 6 and 9 wt.\% of MWCNTs, indicating a percolation threshold within this range. Most recently, Hamedi et al. prepared semitransparent conductive nanopapers from NFC and single-wall carbon nanotubes (SWCNTs) via a similar water based processing route [24]. They proved that carboxylated NFC could successfully exfoliate SWCNTs into individual nanotubes or small bundles and stabilize them in water. They hypothesized that the driving force for the dispersion of SWCNTs by NFC could be an overall gain in entropy combined with the high nonpolar interactions between the two types of nanoparticles. They also showed that the addition of $10 \mathrm{wt} . \%$ SWCNTs led to reduction in modulus, strength and strain-to-failure due to insufficient NFC-SWCNT interactions; nevertheless, the electrical conductivity increased with SWCNT content and reached the highest value of $174 \mathrm{~S} \mathrm{~cm}^{-1}$ at the dispersion limit of this system (43 wt.\% SWCNT).

Hybrid films formed by CNCs and CNTs still need further investigation in terms of CNC-CNT interactions both in aqueous suspensions and solid hybrid films, the hybrid films microstructure and properties. In this study we explore the enhancement effects of CNTs on CNC films and the potential mechanism of CNT reinforcement in the CNC films. The hybrid films made from CNCs and CNTs by a simple vacuum filtration method without the use of surfactants show good mechanical properties and tailorable electrical properties, with potential broad applications in the electronic field. 


\section{Material and methods}

\subsection{Materials}

Microcrystalline cellulose (MCC) (Sigma-Aldrich) in a form of white fine powder with an average particle size of $20 \mu \mathrm{m}$ and a bulk density of $0.5 \mathrm{~g} / \mathrm{cm}^{3}$ at $25{ }^{\circ} \mathrm{C}$ was purchased from Sigma-Aldrich (St. Louis). Multi-walled carbon nanotubes (MWCNTs) (Nanocyl NC7000) with an average diameter of $9.5 \mathrm{~nm}$, an average length of $1.5 \mu \mathrm{m}$ (rendering an average aspect ratio of 158 ) and $90 \%$ carbon purity as reported by the manufacturer were used. As-produced NC7000 MWCNTs are in the form of entangled micro-size aggregates and the MWCNTs were used as received without any pretreatment or purification ("CNTs" is used to refer to NC7000 MWCNTs in the following context). TEMPO (2,2,6,6-tetramethyl-1-piperidinyloxyl, free radical, 98\%, Aldrich), sodium bromide (purity $>99 \%$, Aldrich), sodium hypochlorite solution (reagent grade, available chlorine $10-15 \%$, Aldrich), ethanol (Aldrich) and sodium hydroxide (reagent grade, $\geq 98 \%$, anhydrous, Aldrich) were used for TEMPO-mediated oxidation without any pretreatment. Deionized water was exclusively used in this study. Nitrocellulose filter membranes (Sigma-Aldrich) with pore size of $220 \mathrm{~nm}$ and filter diameter of $47 \mathrm{~mm}$ were used for vacuum filtration.

\subsection{Preparation of Cellulose Nanocrystals}

Cellulose nanocrystals (CNCs) were prepared via TEMPO-mediated oxidation of MCC following a method described by Isogai et al. with some modifications $[15,25]$. Briefly, MCC $2 \mathrm{~g}$ in dry weight was stirred in $200 \mathrm{~mL}$ deionized water where $32 \mathrm{mg}$ TEMPO and $200 \mathrm{mg}$ sodium bromide were already dissolved. Once no large MCC aggregates were observed in the suspension, TEMPO-mediated oxidation was initiated by adding dropwise $7.45 \mathrm{~g}$ of the sodium hypochlorite solution. The $\mathrm{pH}$ of the oxidation 
system was kept at $10.0-10.5$ by adding $0.5 \mathrm{M}$ sodium hydroxide aqueous solution. After oxidation at room temperature under mild magnetic stirring $(\sim 250 \mathrm{rpm})$ for $4 \mathrm{hrs}$, the oxidation reaction was terminated by adding $15 \mathrm{~mL}$ ethanol. The TEMPO-oxidized MCC (O-MCC) was thoroughly washed three times with deionized water and then was dried by a lyophilizer (VirTis BenchTop 4K model) for 3 days. To prepare CNC aqueous suspensions, a certain amount of freeze-dried $\mathrm{O}-\mathrm{MCC}$ was re-dispersed in deionized water and disintegrated to CNCs by ultrasonication (Sonics Materials Vibra Cell VCX500 ultrasonic processor) at 40\% amplitude with a $13 \mathrm{~mm}$ standard probe for $30 \mathrm{~min}$.

\subsection{Film Preparation}

CNC and CNT enhanced CNC hybrid films were prepared in this study. For CNC/CNT hybrid film preparation, a certain amount of CNTs was added in $50 \mathrm{~mL}$ deionized water and the CNT/water mixture was first sonicated for $20 \mathrm{~min}$ by an ultrasonication processor (Sonics Materials Vibra Cell VCX500) at 40\% amplitude with a standard $13 \mathrm{~mm}$ probe; following that, the desired amount of O-MCC was added and the aqueous suspension continued to be sonicated for another 30 min under the same ultrasonication conditions. The hybrid aqueous suspensions were filtrated in a Millipore vacuum filtration assembly with a nitrocellulose filter as schematically shown in Figure 1a. After vacuum filtration, the hybrid nanoparticle wet cakes were dried in a vacuum desiccator for 2 days. A prepared hybrid film is shown in Figure 1b. To prepare CNC films, $300 \mathrm{mg}$ O-MCC was dispersed and disintegrated in $50 \mathrm{~mL}$ deionized water by ultrasonication (30 min, 40\% amplitude and $13 \mathrm{~mm}$ standard probes). Since the CNC size is similar to or less than the filter pore size, the CNC suspension was casted into the film in a crystallizing dish in vacuum oven at $70^{\circ} \mathrm{C}$. Note that the addition of CNTs 
even at $\sim 0.6 \mathrm{wt} . \%$ makes possible to process $\mathrm{CNC}$ films by vacuum filtration. The designation and composition of the films prepared are summarized in Table 1. By controlling the amount of constituent materials, all films prepared have a thickness of around $100 \mu \mathrm{m}$.

\subsection{Characterization}

Thermogravimetric analysis was conducted on a TA TGA Q500 thermogravimetric analyzer from room temperature to $700{ }^{\circ} \mathrm{C}$, under nitrogen atmosphere, at a heating rate of $10^{\circ} \mathrm{C} / \mathrm{min}$. Film composition was determined from the TGA thermographs. An Olympus BX51 microscope was used to observe the morphology of MCC particles before and after TEMPO-mediated oxidation under polarized light. A Veeco diMultiMode V atomic force microscope (Veeco Instruments Inc, USA) with an antimony doped silicon probe (Bruker AFM probe Model: TESP) was used to image the CNCs on silicon wafer and the cryo-microtomed cross sections of hybrid films in tapping mode at room temperature and in an ambient atmosphere. The ranges of resonant frequencies and spring constant for the silicon probe are $366-401 \mathrm{kHz}$ and 20 $-80 \mathrm{~N} / \mathrm{m}$, respectively. Very dilute CNC suspensions (less than $0.1 \mathrm{mg} / \mathrm{ml}$ ) were prepared for probing particle size and size distribution by AFM. The CNC concentration is sufficiently low to ensure that most of the CNC particles are oriented in the silicon wafer plane. Film tension tests were performed on a Zwick-Roell Z0.5 tensile tester equipped with a $100 \mathrm{~N}$ load cell at an extension speed of $1 \mathrm{~mm} / \mathrm{min}$ at room temperature and a relative humidity of around $40 \%$. The tensile samples were cut from the prepared films into a rectangular shape (20 $\mathrm{mm}$ length and $4 \mathrm{~mm}$ width $)$ and at least 5 specimens were measured for each sample. Scanning electron microscopy (SEM) was performed on a Helios Nanolab 650 field emission electron microscope on sputter 
coated samples with an acceleration voltage of $2 \mathrm{kV}$ and $13 \mathrm{pA}$ beam current for the hybrid films. The BET surface areas $(A B E T)$ of the films were measured by a Micromeritics TriStar II surface area and porosity analyzer with nitrogen as adsorptive at $77 \mathrm{~K}$ up to a relative pressure $\left(P / P_{0}\right)$ of around 0.2189 . Since the $P / P_{0}$ is below 0.4 , the specific area measured is from the external surface of CNTs rather than the internal surface [26]. The porosity of the CNC film was measured according to a density method by using a Mettler Toledo analytical balance with ethanol as immersing solvent at room temperature [27]. Electrical conductivity was measured with an 11-point $d c$ surface resistance meter (PRS-801, Prostat) equipped with a miniature concentric ring fixture (PRF-912B). The PRF-912B resistance fixture approximates the same measurements obtained when using a standard ESD Association Standard ANSI/ESD S11.11 concentric ring fixture. Following the ESD standard, to convert a resistance obtained by this method to an equivalent resistivity in ohms per square, the resistance measurements were multiplied by ten. The conversion factor of ten is derived from the geometry of the electrode assembly. Measurements were repeated on at least 6 different areas of the film to ensure sample uniformity and their geometric average are reported. Different relative humidity levels were generated by saturated salt solutions in a sealed small chamber according to ASTM E104 standard using potassium acetate, sodium bromide and potassium chloride.

\section{Results and Discussion}

\subsection{CNC Films}

TEMPO-mediated oxidation converts primary hydroxyl groups on the surface of cellulose crystallites into carboxylate groups as well as degrades the links between cellulose crystallites leading to the easy disintegration of oxidized MCC (O-MCC) into 
CNCs by mechanical forces. Polarized optical microscopy images in Figure 2 ( $a \& b)$ illustrate the MCC morphology change from intact to irregular after TEMPO-mediated oxidation. The O-MCC was further disintegrated by ultrasonication into CNCs which could not be observed with optical microscopy. Atomic force microscopy was used to image nano-sized CNC particles as shown in Figure 2 (c, $d \&$ e). The height and phase images of the $\mathrm{CNCs}$ show rod-like $\mathrm{CNC}$ particles and a height of around $5 \mathrm{~nm}$ for individual CNC particles. Two hundred forty (240) individual CNCs were identified in multiple AFM height images in order to measure their heights and lengths from which the length distribution of the $\mathrm{CNC}$ particles can be obtained (Figure 2f). The length distribution generally follows a Gaussian distribution indicating that the number of CNC particle counted is adequate for statistical analysis. The average length and height of the CNCs are 258.9 and $4.3 \mathrm{~nm}$, respectively, leading to an average aspect ratio of 60 . As compared to the dimensions of the MWCNTs used in this study (average diameter of $9.5 \mathrm{~nm}$, an average length of $1.5 \mu \mathrm{m}$, an average aspect ratio of 158), the CNCs are much shorter and have lower aspect ratio. The discernible dimension difference between the CNTs and CNCs could be one of the reasons for the improved mechanical properties and interesting micro-structure of CNT enhanced CNC films. The CNCs are negatively charged in water due to the presence of carboxylate groups and thus can be well dispersed in water with the electrostatic repulsion keeping the dispersion stable [14].

CNC films were fabricated via solvent casting, as the length of the CNCs is comparable with the pore size of the filter membranes which precludes membrane filtration. The addition of as low as $\sim 0.6 \mathrm{wt} . \%$ CNTs allows the CNCs to be processed by vacuum filtration into films because the CNTs could block filter membrane pores 
leading to reduced pore size. $\mathrm{CNC}$ particles assemble layer-by-layer during solvent casting with the axial direction of the CNCs parallel to the radial direction of the final films as proved by the SEM images in Figure $3(\mathrm{a} \& \mathrm{~b})$. The pull-out of CNC layers during tensile testing leaves string like dents on the fracture surface (Figure $3 \mathrm{~b}$ ). Tensile failure of the $\mathrm{CNC}$ films is mainly due to the $\mathrm{CNC}$ interparticle hydrogen bond breakage which explains the relatively low mechanical properties of the $\mathrm{CNC}$ films as compared to single $\mathrm{CNC}$ particles $[28,29]$. The $\mathrm{CNC}$ films have tensile modulus, strength and toughness of $3.89 \mathrm{GPa}, 113.6 \mathrm{MPa}$ and $1.78 \times 10^{6} \mathrm{~J} / \mathrm{m}^{3}$, respectively. Because of the linear rod-like morphology, nano-scale size, and interparticle hydrogen bonds, $\mathrm{CNC}$ particles tend to pack tightly during film formation resulting in a very low $A_{B E T}$ value of $2.12 \mathrm{~m}^{2} / \mathrm{g}$. The high surface electrical resistivity $\left(2.8 \times 10^{11} \Omega / \mathrm{sq}\right)$ is inline with the electrical insulation properties of cellulosic materials.

\subsection{Morphology and Tensile Properties of the CNT enhanced CNC Films}

$\mathrm{CNC}$ and $\mathrm{CNT}$ particles in aqueous suspensions self-assemble into free-standing CNC/CNT hybrid films by van der Waals forces between CNT particles, hydrogen bonds between $\mathrm{CNC}$ particles and potential $\pi$-cation interaction between the $\mathrm{CNT}$ and CNC particles [30, 31]. Figure 3 (c \& d) illustrates the morphology of the tensile fracture surfaces of the CNT enhanced CNC films containing $1.2 \mathrm{wt}$ \% CNTs (CNTC1.2\%). Larger dents and layered protrusions are observed on the fracture surfaces of the CNTC- $1.2 \%$ film by comparison with the relatively smooth fracture surfaces of the CNC films. Clearly seen on these micrographs are CNT pull-outs (indicated by the red arrows) as well as CNT reinforced CNC protruded layers. Thus the fracture mechanism for the $\mathrm{CNT}$ enhanced $\mathrm{CNC}$ films changes from only hydrogen bond breakage for the 
CNC film to CNT pulling and hydrogen bond breakage between CNT reinforced CNC layers.

Figure 4 illustrates the trends of tensile properties of the CNT enhanced CNC films with CNT weight fraction. In Figure 4a both tensile modulus and strength increase rapidly when adding only a small amount of CNTs (1.2 and $2.2 \mathrm{wt} . \%)$ which reveals the immediate reinforcement effect of CNTs in the CNC films. With further increasing CNT weight fraction to 5.2, 10.2 and $11.6 \mathrm{wt} . \%$, the tensile modulus and strength do not show a smooth monotonic increment trend, however considering the error bars it could be stated that the tensile modulus and strength reach peak values $(6.26 \pm 0.27 \mathrm{GPa}$ for modulus and $179.7 \pm 4.1 \mathrm{MPa}$ for strength) at around 10.2 to $11.6 \mathrm{wt} . \% \mathrm{CNT}$. The small tensile testing bars were manually cut from films, and although at least 5 specimens were measured for each data point, large error bars could not be eluded due to the variation of film flatness, thickness and cutting edge smoothness as well as tiny deviation from vertical alignment during clamping sample bars.

The tensile properties are considered to be influenced by the balance between the CNT dispersion state and the extent of forming a continuous CNT network. As the CNT concentration increases from 2.2 to $10.2 \mathrm{wt} \%$, the potential of forming CNT aggregates/bundles increases but meanwhile a more complete CNT continuous network is forming. Further increase in the CNT weight fraction to $20.1 \mathrm{wt} . \%$ causes the tensile modulus and strength to decrease to $5.46 \pm 0.18 \mathrm{GPa}$ and $143.0 \pm 15.2 \mathrm{MPa}$. We have also prepared a hybrid film with $62 \mathrm{wt} . \%$ CNTs and found further decrease in mechanical properties: the modulus dropped to $2.95 \pm 0.11 \mathrm{GPa}$ and the strength to $46.9 \pm 3.2 \mathrm{MPa}$. As the $\mathrm{CNT}$ concentration exceeds a critical value, i.e. 10.2 to 11.6 wt.\% in this study, the CNTs will form more aggregates/bundles and the voids between 
the CNTs could not be completely filled by CNCs resulting in an overall decrease in the mechanical properties. The $A_{B E T}$ measurement proves this hypothesis: the hybrid films containing up to 20.1 wt. $\%$ CNTs show $\mathrm{A}_{B E T}$ values close to $0 \mathrm{~m}^{2} / \mathrm{g}\left(0.026 \mathrm{~m}^{2} / \mathrm{g}\right.$ for the 20.1 wt.\% CNTs) but this value increses dramatically at higher CNT content (135.9 $\mathrm{m}^{2} / \mathrm{g}$ at $62 \mathrm{wt} . \% \mathrm{CNTs}$ ) indicating a large increase in voids located within CNT bundles leading to reduced tensile properties. On the other hand, at too low CNT concentrations, the CNTs are not able to form a well percolated network resulting in minor improvements in tensile properties.

Previous reports $[23,24]$ do not show such improvement in mechanical properties of nanofibrillated cellulose (NFC)/ CNT hybrid films, mainly due to the film high porosity caused by the long (several micrometers) NFCs employed in the fabrication.

Figure $4 \mathrm{~b}$ plots the stress-strain curves for the CNC and CNT enhanced CNC films at different CNT concentrations. The CNC film exhibits a linear stress-strain behavior typical for a brittle film due to the rigid nature of $\mathrm{CNC}$ particles and the short range of hydrogen bond interactions. With CNT addition the behavior changes toward a more ductile response and increased toughness. The tensile toughness of the hybrid films is plotted in Figure 4c as a function of CNT concentration. The CNT enhanced CNC films show an increase in toughness as high as about 3 times by comparison with the CNC films. The tensile toughness shows the same trend as the tensile modulus and strength. Interestingly, the $\mathrm{CNT}$ enhanced $\mathrm{CNC}$ films also tend to approach a maximum tensile toughness at a CNT weight fraction around $10 \%$.

The improvements in tensile properties for the CNT enhanced CNC films as compared to $\mathrm{CNC}$ films are potentially due to synergistic effects between the two nanofillers. In aqueous suspensions, CNCs help dispersing and stabilizing CNTs and 
CNTs in turn allow the process of CNCs into films by vacuum filtration. Cryomicrotomed cross sections of films were analyzed by AFM. Figure 5 shows AFM phase images of the cross section for the 10.2 wt.\% CNT reinforced CNC film. The lighter and brighter spots on the AFM phase images represent the cut ends of the CNTs. The size of the spots is around $9-12 \mathrm{~nm}$ which corresponds to the diameter of the CNTs used. The spots are uniformly dispersed on the cross sections and big bundles are barely observed, pointing out to good CNT dispersion in the CNC matrix. In solid hybrid films, CNTs distributed between CNC layers could form a continuous network at a critical concentration $\left(\omega_{c}\right)$. This $\omega_{c}$ is determined by the dimensions as well as the dispersion state of the CNTs. Based on the tensile properties results, this critical CNT concentration for the system investigated is in the range between 10.2 to $11.6 \mathrm{wt}$ \% $\%$. At this concentration CNTs form a continuous network by CNT-CNT junctions and entanglement points which are protected by tightly packed CNC matrix. The CNT network constrains the $\mathrm{CNC}$ matrix deformation which explains the increase in tensile modulus. At the same time, the junction and entanglement points in the CNT network are protected by a tightly packed $\mathrm{CNC}$ matrix which adds to the system toughness; at this point, the smaller size of the rod-like CNC as compared to the CNTs assures the access to the voids among the CNTs and the tight packing. Below $\omega_{c}$, CNTs can't form a continuous network although some of the CNTs could be entangled with each other. Above $\omega_{c}$, the CNTs also form a continuous network but the amount of CNCs in the system is no longer sufficient to fill all the voids between the CNT entanglements and CNT aggregates/bundles tend to form. Right at the $\omega_{c}$, the $\mathrm{CNC} / \mathrm{CNT}$ hybrid films, compared to CNT reinforced polymeric composite systems, have two main advantages: first, the CNTs could be uniformly dispersed in CNC aqueous suspensions; second, the 
CNT enhanced CNC films show superior mechanical properties by comparison with most conventional polymers and respective films [32].

\subsection{CNT enhanced CNC Films with Tunable Electrical Properties}

Figure 6 presents the surface electrical resistivity of $\mathrm{CNC}$ and $\mathrm{CNT}$ enhanced $\mathrm{CNC}$ films at three relative humidity levels $(22 \%, 59 \%$ and $85 \%)$. The $\mathrm{CNC}$ films are characterized by a surface electrical resistivity of about $2.8 \times 10^{11} \Omega / \mathrm{sq}$ at a relative humidity of about $22 \%$ which decreases 5 orders of magnitude at $85 \%$ humidity. The CNT enhanced CNC films show a wide range of surface electrical resistivities from more than $10^{11}$ to about $10^{2} \Omega /$ sq. Based on surface electrical resistivity, materials can be classified as metals $\left(10^{-5}\right.$ to $\left.10^{-1} \Omega / \mathrm{sq}\right)$, conductive materials $\left(10^{1}\right.$ to $\left.10^{6} \Omega / \mathrm{sq}\right)$, static dissipative materials $\left(10^{6}\right.$ to $\left.10^{12} \Omega / \mathrm{sq}\right)$ and plastics $\left(10^{12} \&\right.$ up $\left.\Omega / \mathrm{sq}\right)$ [33]. Based on the classification, the CNT enhanced $\mathrm{CNC}$ films can be used as conductive and static dissipative materials. At 5 wt.\% CNTs, the surface resistivity of the CNT enhanced CNC films drops and is not affected by humidity. The CNT enhanced CNC films show surface resistivity highly sensitive to the environment relative humidity at CNT weight fraction less than $5 \%$, which opens the possibility of using such films to replace resistive type humidity sensors made from polymer thin films with advantages such as low cost and durability [34].

\section{Conclusions}

In this study commercial MWCNTs and bio-based CNCs were used to prepare CNT enhanced $\mathrm{CNC}$ films via an environmental friendly and simple aqueous suspension vacuum filtration method. The $\mathrm{CNT}$ enhanced $\mathrm{CNC}$ films were compared with $\mathrm{CNC}$ films showing promising tensile and electrical properties. The addition of CNTs allows processing $\mathrm{CNC}$ films by a vacuum filtration method which is more convenient than the 
solvent casting method. The CNT enhanced CNC films, prepared from water suspensions made without surfactant, show good mechanical and electrical properties, reflective of the dispersing effect of CNCs on CNTs in aqueous suspensions. The tensile properties of these films suggest the formation of a continuous CNT network at CNT concentration levels in the range between 10.2 - 11.6 wt.\% CNT. These CNT enhanced $\mathrm{CNC}$ films exhibit a more ductile behavior by comparison with the $\mathrm{CNC}$ film, rendering an increase in toughness. The improved mechanical properties are believed to be due to the synergistic effects between CNCs and CNTs in the hybrid films, derived from the protection of junction and entanglement points in the continuous CNT network by the closely packed CNC matrix. Layer-by-layer assembly of CNCs and the dispersion of individualized CNTs in CNC matrix are confirmed by the FE-SEM and AFM micrographs. The surface electrical resistivity for the hybrid films within a wide range from $10^{2}$ to $10^{11} \Omega /$ sq can be tailored by manipulating the CNT weight fraction, which opens a wide window for the application of these hybrid films in the electronic field. Moreover, the humidity sensitive surface electrical resistivity makes these hybrid films a viable candidate for sensor applications.

\section{Acknowledgements}

We acknowledge the National Science Foundation for the financial support of our research through grant PIRE-1243313. Acknowledgements are also due to Vahab

Solouki Bonab and Liang Yue for their help with some experimental work.

\section{References}

1. Xia, Y., P. Yang, Y. Sun, Y. Wu, B. Mayers, B. Gates, Y. Yin, F. Kim, and H. Yan, One-Dimensional Nanostructures: Synthesis, Characterization, and Applications. Advanced Materials, 2003. 15(5): p. 353-389.

2. Eletskii, A.V. and B.M. Smirnov, Fullerenes and carbon structures. PhysicsUspekhi, 1995. 38(9): p. 935. 
3. Wong, E.W., P.E. Sheehan, and C.M. Lieber, Nanobeam Mechanics: Elasticity, Strength, and Toughness of Nanorods and Nanotubes. Science, 1997. 277(5334): p. 1971-1975.

4. Baughman, R.H., A.A. Zakhidov, and W.A. de Heer, Carbon nanotubes - the route toward applications. Science, 2002. 297(5582): p. 787-792.

5. Liang, W.J., M. Bockrath, D. Bozovic, J.H. Hafner, M. Tinkham, and H. Park, Fabry-Perot interference in a nanotube electron waveguide. Nature, 2001. 411(6838): p. 665-669.

6. Frank, S., P. Poncharal, Z.L. Wang, and W.A.d. Heer, Carbon Nanotube Quantum Resistors. Science, 1998. 280(5370): p. 1744-1746.

7. Kim, P., L. Shi, A. Majumdar, and P.L. McEuen, Thermal Transport Measurements of Individual Multiwalled Nanotubes. Physical Review Letters, 2001. 87(21): p. 215502.

8. Tashiro, K. and M. Kobayashi, Theoretical evaluation of three-dimensional elastic constants of native and regenerated celluloses: role of hydrogen bonds. Polymer, 1991. 32(8): p. 1516-1526.

9. Sakurada, I., Y. Nukushina, and T. Ito, Experimental determination of the elastic modulus of crystalline regions in oriented polymers. Journal of Polymer Science, 1962. 57(165): p. 651-660.

10. Diddens, I., B. Murphy, M. Krisch, and M. Müller, Anisotropic Elastic Properties of Cellulose Measured Using Inelastic X-ray Scattering. Macromolecules, 2008. 41(24): p. 9755-9759.

11. Šturcová, A., G.R. Davies, and S.J. Eichhorn, Elastic Modulus and StressTransfer Properties of Tunicate Cellulose Whiskers. Biomacromolecules, 2005. 6(2): p. 1055-1061.

12. Rusli, R. and S.J. Eichhorn, Determination of the stiffness of cellulose nanowhiskers and the fiber-matrix interface in a nanocomposite using Raman spectroscopy. Applied Physics Letters, 2008. 93(3): p. -.

13. Iwamoto, S., W. Kai, A. Isogai, and T. Iwata, Elastic Modulus of Single Cellulose Microfibrils from Tunicate Measured by Atomic Force Microscopy. Biomacromolecules, 2009. 10(9): p. 2571-2576.

14. Capadona, J.R., K. Shanmuganathan, S. Trittschuh, S. Seidel, S.J. Rowan, and C. Weder, Polymer Nanocomposites with Nanowhiskers Isolated from Microcrystalline Cellulose. Biomacromolecules, 2009. 10(4): p. 712-716.

15. Isogai, A., T. Saito, and H. Fukuzumi, TEMPO-oxidized cellulose nanofibers. Nanoscale, 2011. 3(1): p. 71-85.

16. Sobkowicz, M.J., B. Braun, and J.R. Dorgan, Decorating in green: surface esterification of carbon and cellulosic nanoparticles. Green Chemistry, 2009. 11(5): p. 680-682.

17. Braun, B. and J.R. Dorgan, Single-Step Method for the Isolation and Surface Functionalization of Cellulosic Nanowhiskers. Biomacromolecules, 2009. 10(2): p. 334-341. 
18. Oksman, K., J.A. Etang, A.P. Mathew, and M. Jonoobi, Cellulose nanowhiskers separated from a bio-residue from wood bioethanol production. Biomass and Bioenergy, 2011.35(1): p. 146-152.

19. Fukuzumi, H., T. Saito, T. Iwata, Y. Kumamoto, and A. Isogai, Transparent and High Gas Barrier Films of Cellulose Nanofibers Prepared by TEMPO-Mediated Oxidation. Biomacromolecules, 2008. 10(1): p. 162-165.

20. Chun, S.-J., S.-Y. Lee, G.-H. Doh, S. Lee, and J.H. Kim, Preparation of ultrastrength nanopapers using cellulose nanofibrils. Journal of Industrial and Engineering Chemistry, 2011. 17(3): p. 521-526.

21. Liu, A., A. Walther, O. Ikkala, L. Belova, and L.A. Berglund, Clay Nanopaper with Tough Cellulose Nanofiber Matrix for Fire Retardancy and Gas Barrier Functions. Biomacromolecules, 2011. 12(3): p. 633-641.

22. Fugetsu, B., E. Sano, M. Sunada, Y. Sambongi, T. Shibuya, X. Wang, and T. Hiraki, Electrical conductivity and electromagnetic interference shielding efficiency of carbon nanotube/cellulose composite paper. Carbon, 2008. 46(9): p. 1256-1258.

23. Salajkova, M., L. Valentini, Q. Zhou, and L.A. Berglund, Tough nanopaper structures based on cellulose nanofibers and carbon nanotubes. Composites Science and Technology, 2013. 87: p. 103-110.

24. Hamedi, M.M., A. Hajian, A.B. Fall, K. Håkansson, M. Salajkova, F. Lundell, L. Wågberg, and L.A. Berglund, Highly Conducting, Strong Nanocomposites Based on Nanocellulose-Assisted Aqueous Dispersions of Single-Wall Carbon Nanotubes. ACS Nano, 2014.

25. Habibi, Y., H. Chanzy, and M. Vignon, TEMPO-mediated surface oxidation of cellulose whiskers. Cellulose, 2006. 13(6): p. 679-687.

26. Inoue, S., N. Ichikuni, T. Suzuki, T. Uematsu, and K. Kaneko, Capillary Condensation of N2 on Multiwall Carbon Nanotubes. The Journal of Physical Chemistry B, 1998. 102(24): p. 4689-4692.

27. Mwaikambo, L.Y. and M.P. Ansell, The determination of porosity and cellulose content of plant fibers by density methods. Journal of Materials Science Letters, 2001. 20(23): p. 2095-2096.

28. Kontturi, E., T. Tammelin, and M. Osterberg, Cellulose-model films and the fundamental approach. Chemical Society Reviews, 2006. 35(12): p. 1287-1304.

29. Kulachenko, A., T. Denoyelle, S. Galland, and S.B. Lindström, Elastic properties of cellulose nanopaper. Cellulose, 2012. 19(3): p. 793-807.

30. Ma, J., J.-N. Wang, C.-J. Tsai, R. Nussinov, and B. Ma, Diameters of singlewalled carbon nanotubes (SWCNTS) and related nanochemistry and nanobiology. Frontiers of Materials Science in China, 2010. 4(1): p. 17-28.

31. Ratna, D., T. N. Abraham, S. Siengchin, and J. Karger-Kocsis, Novel Method for Dispersion of Multiwall Carbon Nanotubes in Poly(ethylene oxide) Matrix Using Dicarboxylic Acid Salts. Journal of Polymer Science: Part B: Polymer Physics, 47 (12): p. 1156-1165. 
32. Kalpakjian, S. and S. Schmid, Manufacturing Processes for Engineering Materials. 2009, Reading, MA, USA: Addison-Wesley.

33. Al-Saleh, M.H. and U. Sundararaj, A review of vapor grown carbon nanofiber/polymer conductive composites. Carbon, 2009. 47(1): p. 2-22.

34. Sakai, Y., Y. Sadaoka, and M. Matsuguchi, Humidity sensors based on polymer thin films. Sensors and Actuators B: Chemical, 1996. 35(1-3): p. 85-90.
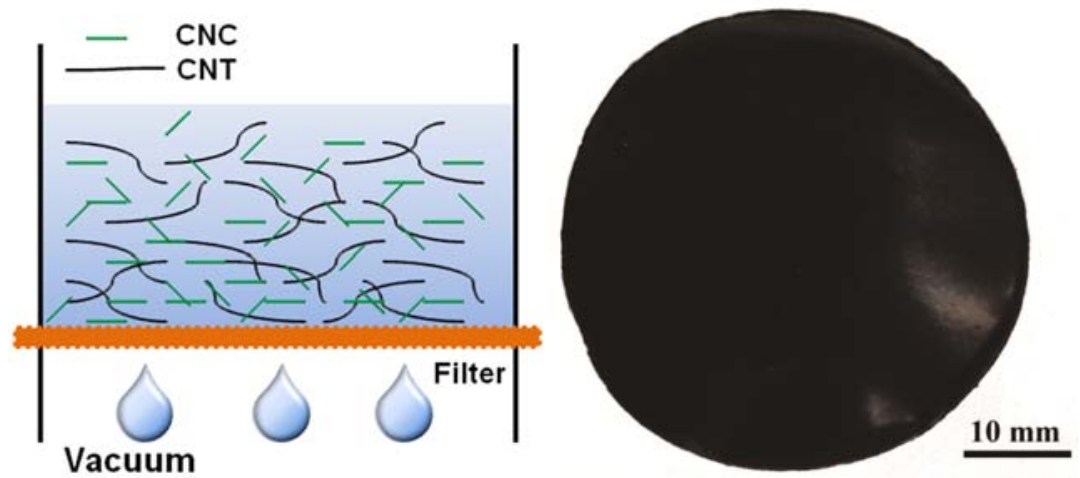

Fig. 1. Schematic of vacuum filtration of $\mathrm{CNC} / \mathrm{CNT}$ aqueous suspension (a) and a photo image of a CNT enhanced CNC film (b).
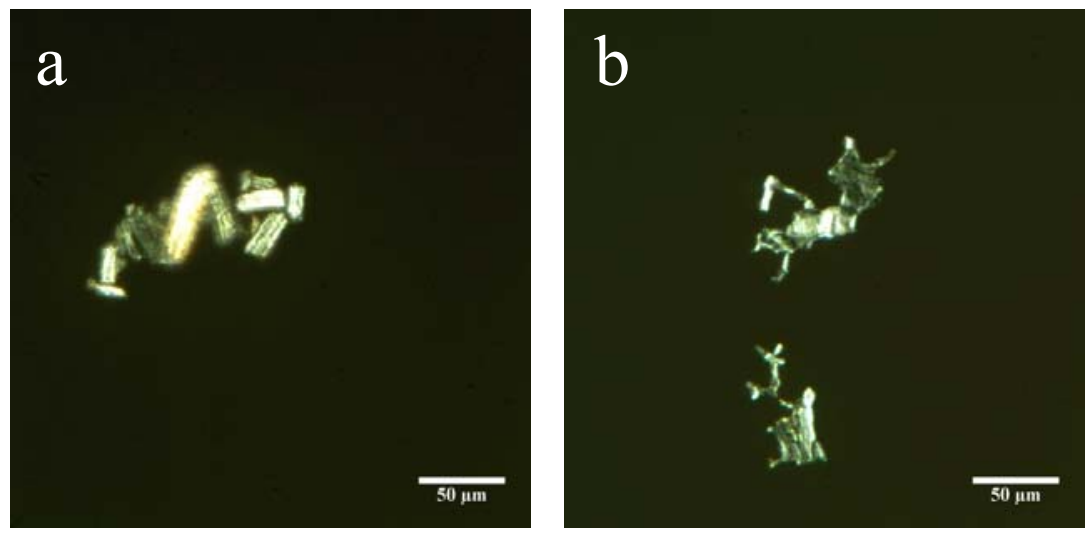

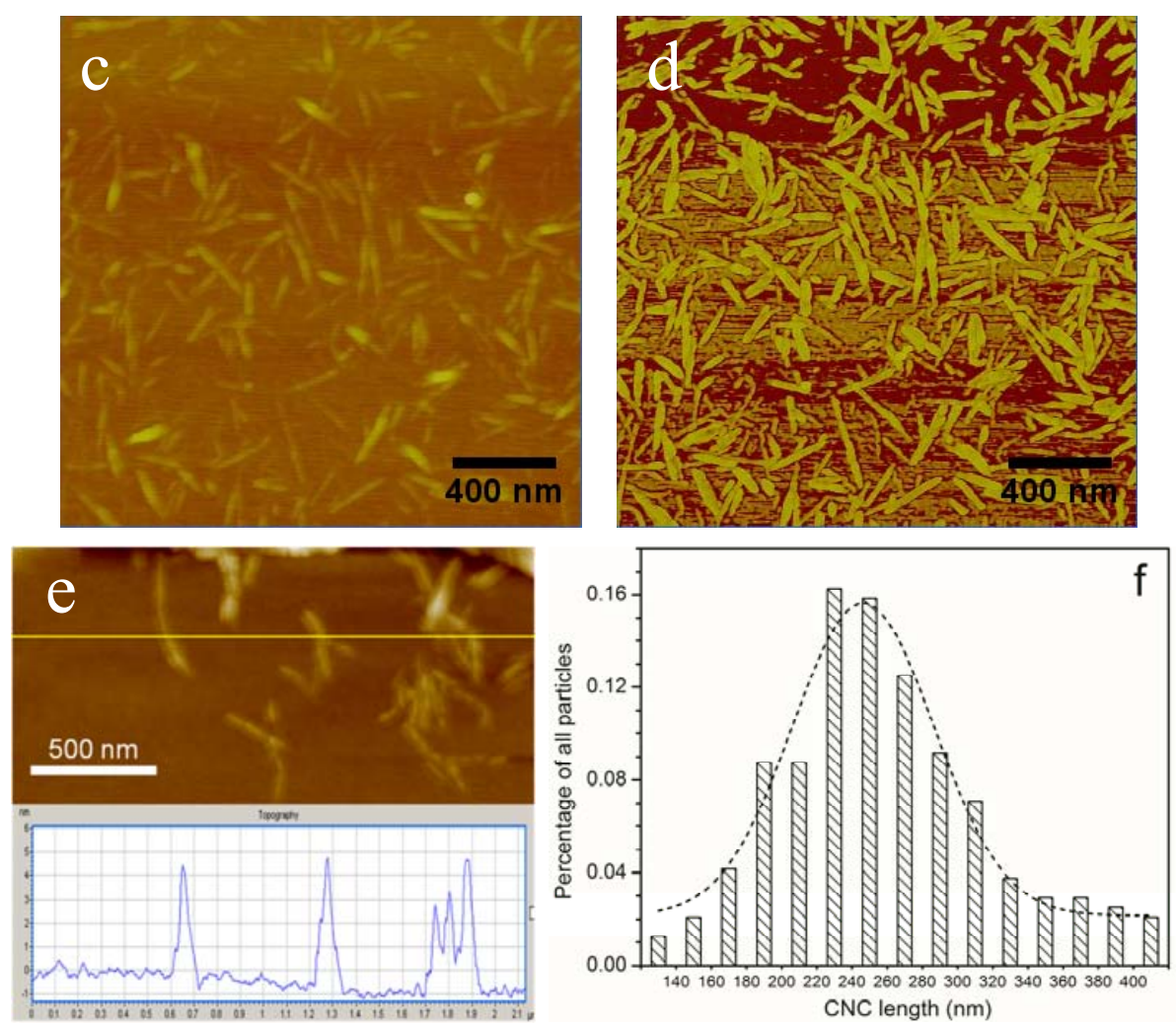

Fig. 2. Polarized optical microscopy images of MCC before (a) and after (b) TEMPOmediated oxidation and AFM images of cellulose nanocrystals (c) height and (d) phase, (e) height graph of CNCs with topography (f) and cellulose nanocrystal length distribution.
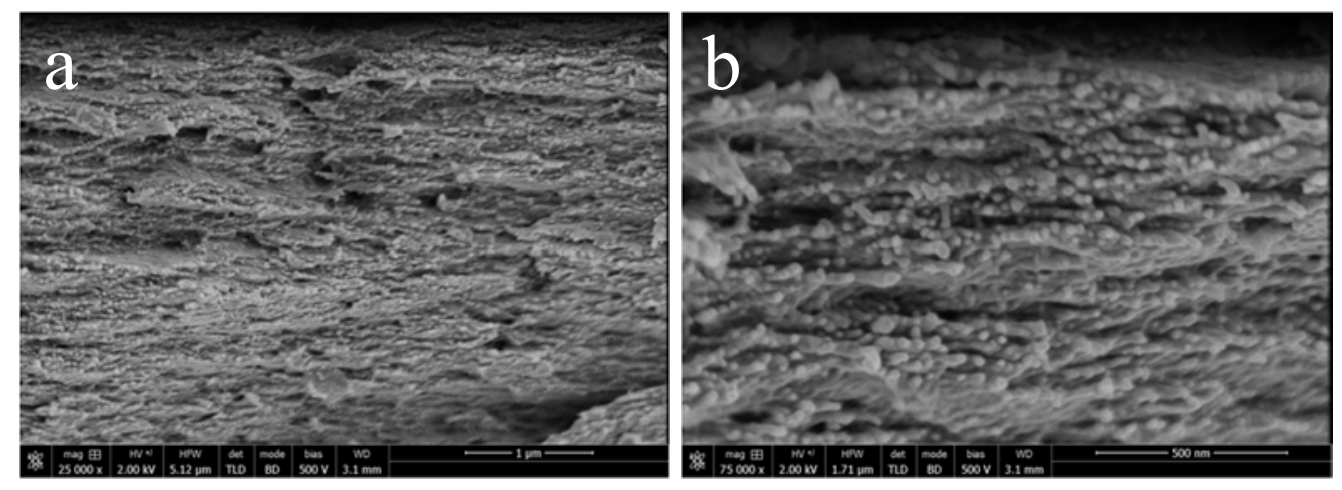

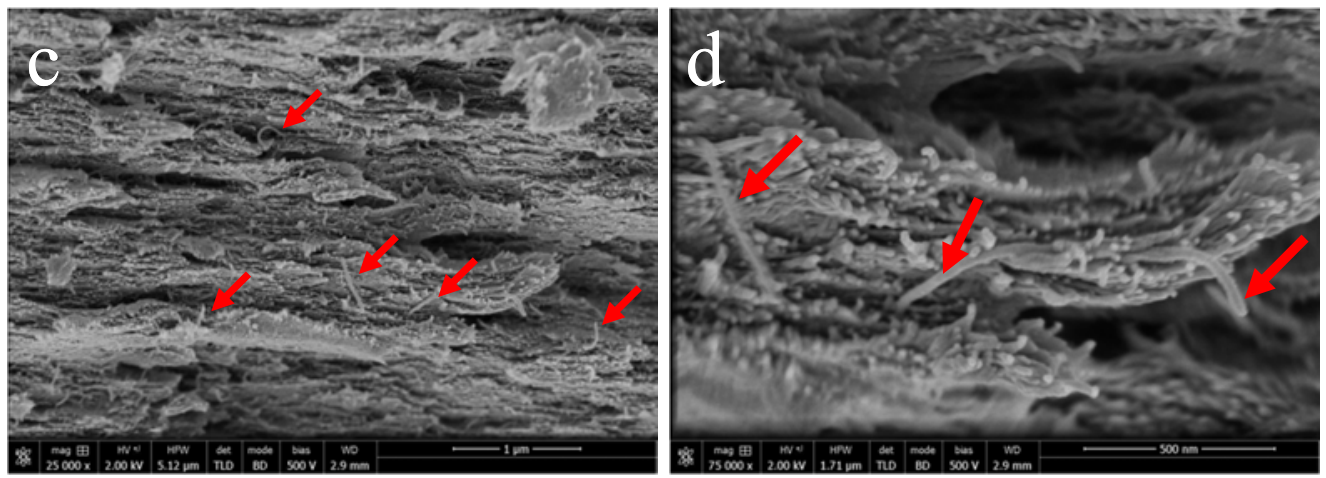

Fig. 3. FE-SEM micrographs of fracture surfaces of CNC ( $\mathrm{a} \& \mathrm{~b})$ and $1.2 \mathrm{wt} \% \mathrm{CNT}$ enhanced $\mathrm{CNC}$ (c \& d) films at two magnifications: $25000 \mathrm{X}$ and $75000 \mathrm{X}$.
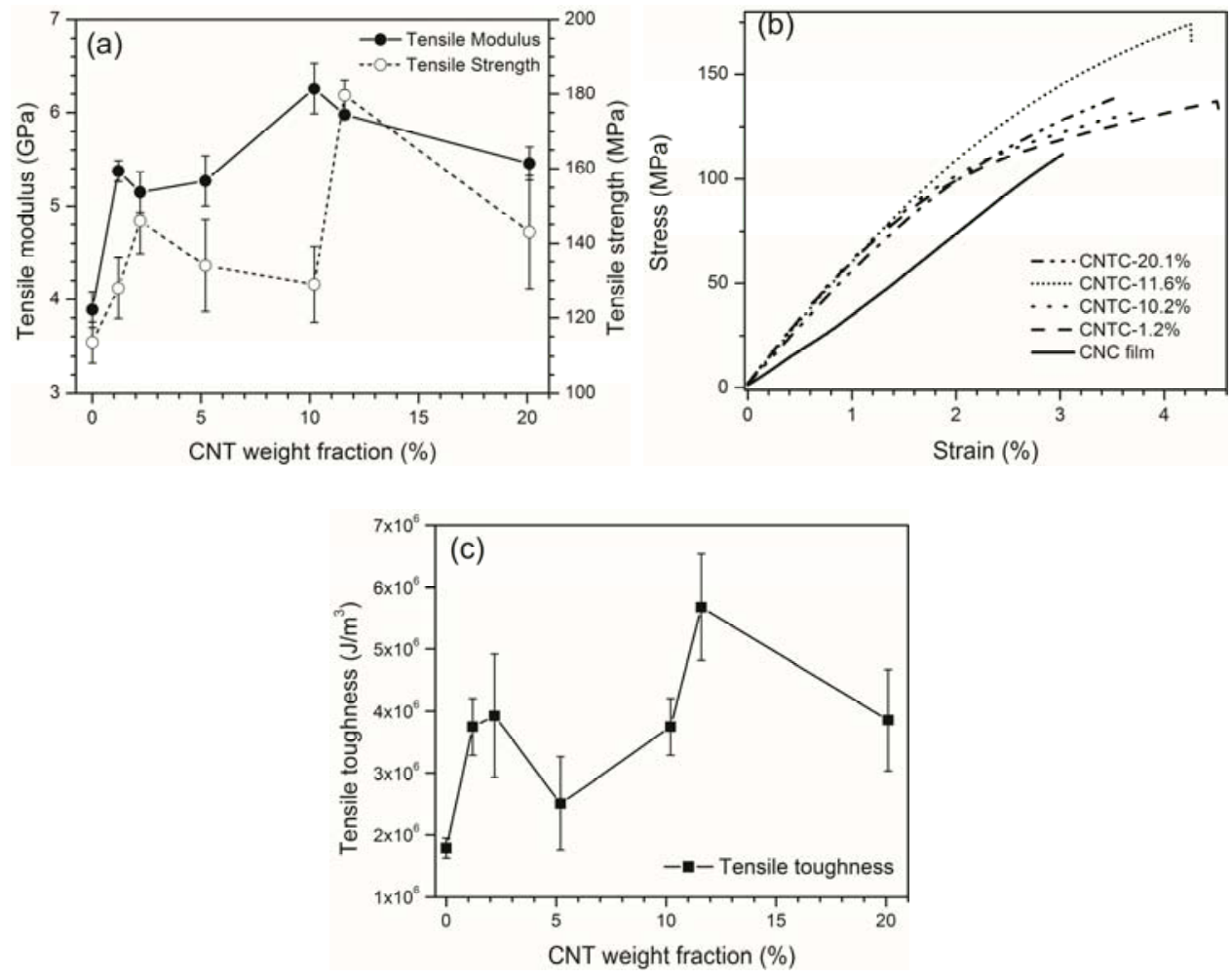

Fig. 4. Tensile properties of the CNT enhanced CNC films as a function of CNT weight fraction: (a) tensile modulus and strength; (b) stress-strain behaviors of CNC and CNT enhanced $\mathrm{CNC}$ films and (c) tensile toughness. 

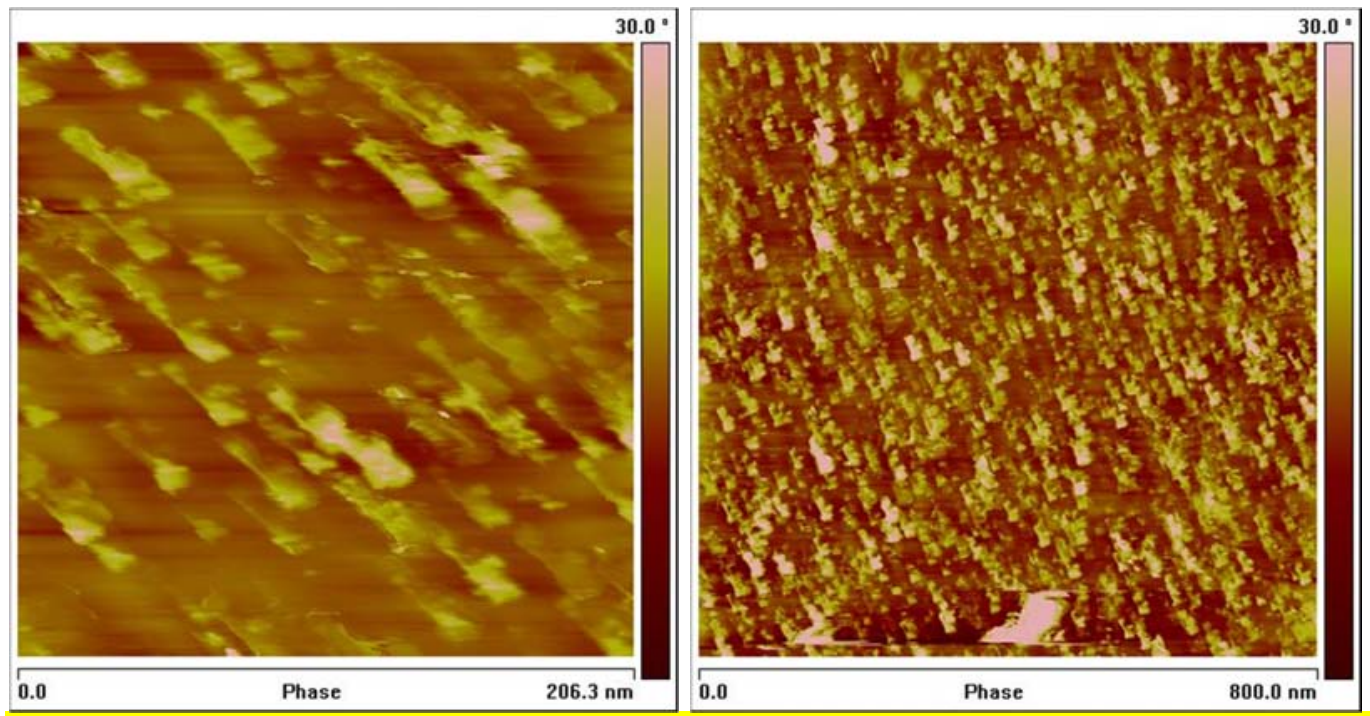

Fig. 5. AFM phase images of the cryo-microtomed cross sections of the $10.2 \mathrm{wt} . \% \mathrm{CNT}$ reinforced $\mathrm{CNC}$ film at two different magnifications

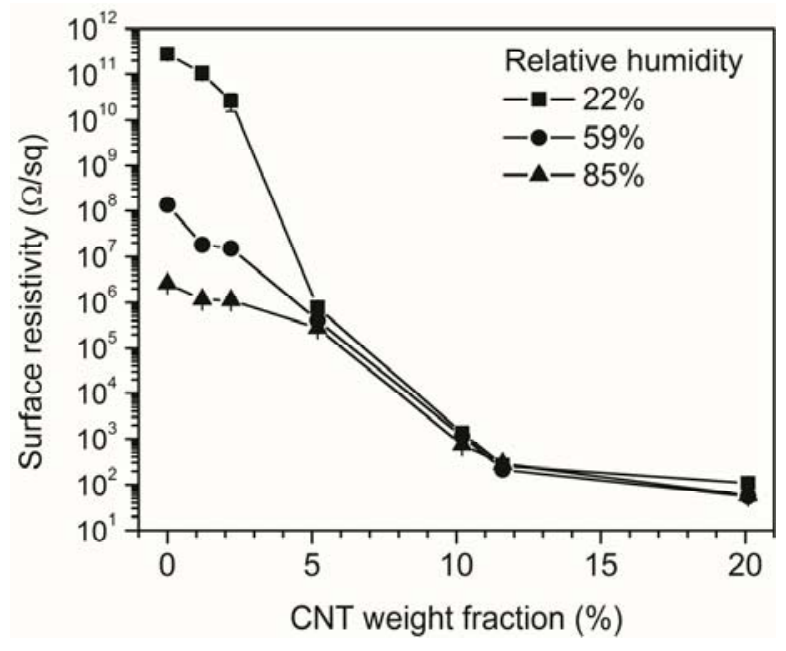

Fig. 6. Surface electrical resistivity of CNT enhanced CNC films as a function of CNT weight fraction at three relative humidity levels $(22 \%, 59 \%$ and $85 \%)$.

\section{Figure Captions}

Fig. 1. Schematic of vacuum filtration of $\mathrm{CNC} / \mathrm{CNT}$ aqueous suspension (a) and a photo image of a CNT enhanced CNC film (b).

Fig. 2. Polarized optical microscopy images of MCC before (a) and after (b) TEMPOmediated oxidation and AFM images of cellulose nanocrystals (c) height and (d) phase, 
(e) height graph of CNCs with topography (f) and cellulose nanocrystal length distribution.

Fig. 3. FE-SEM micrographs of fracture surfaces of CNC (a \& b) and $1.2 \mathrm{wt} \% \mathrm{CNT}$ enhanced CNC (c \& d) films at two magnifications: $25000 \mathrm{X}$ and $75000 \mathrm{X}$.

Fig. 4. Tensile properties of the CNT enhanced CNC films as a function of CNT weight fraction: (a) tensile modulus and strength; (b) stress-strain behaviors of CNC and CNT enhanced $\mathrm{CNC}$ films and (c) tensile toughness.

Fig. 5. AFM phase images of the cryo-microtomed cross sections of the $10.2 \mathrm{wt} . \% \mathrm{CNT}$ reinforced $\mathrm{CNC}$ film at two different magnifications.

Fig. 6. Surface electrical resistivity of CNT enhanced CNC films as a function of CNT weight fraction at three relative humidity levels $(22 \%, 59 \%$ and $85 \%)$.

\section{Table 1}

Designation and composition of the films prepared

\begin{tabular}{ccc}
\hline Film designation & CNTs [wt. \%] & CNCs [wt. \%] \\
\hline CNC film & 0 & 100 \\
CNTC-1.2\% & 1.2 & 98.8 \\
CNTC-2.2\% & 2.2 & 97.8 \\
CNTC-5.2\% & 5.2 & 94.8 \\
CNTC-10.2\% & 10.2 & 89.8 \\
CNTC-11.6\% & 11.6 & 88.4 \\
CNTC-20.1\% & 20.1 & 79.9 \\
\hline
\end{tabular}

